\title{
Les générateurs photovoltaïques autonomes sans batteries
}

\author{
J. A. Roger, J. Pivot \\ Département de Physique des Matériaux $\left(^{*}\right)$ \\ I 'niversité Claude-Bernard, Lyon I, 43, bd 11-Novembre-1918, 69622 Villeurbanne, France \\ et P. Gucher \\ Institut de Chimie et de Physique Industrielles, 31, place Bellecour, 69002 Lyon, France
}

(Reçu le 27 juillet 1979, révisé le 10 décembre 1979, accepté le 12 décembre 1979)

\begin{abstract}
Résumé. - Après une très brève présentation des différentes conceptions de générateurs photovoltaïques autonomes, cet article aborde le cas des systèmes sans stockage d'énergie électrique alimentant des charges du type continu. On envisage successivement :

- le couplage direct entre panneaux et moteur continu,

- la liaison par adaptateur' de charge.

L'article souligne l'intérêt et les limites de chacune de ces techniques en tenant compte de considérations liées à l'exploitation.
\end{abstract}

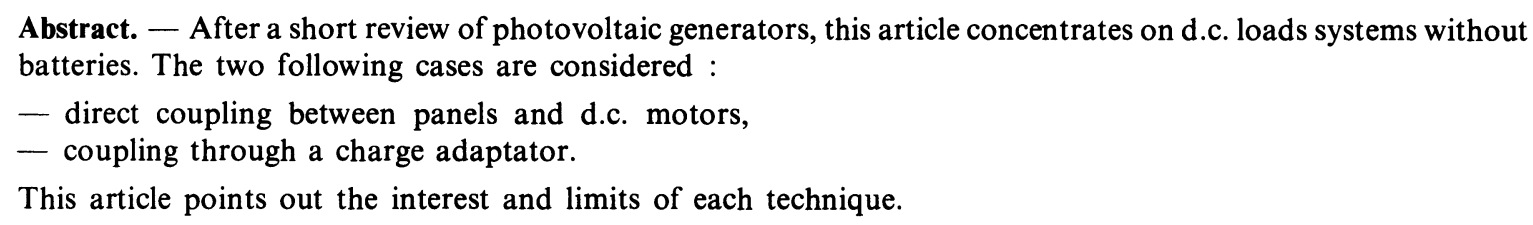

1. Introduction. - Les générateurs photovoltaïques, qui ont connu une période d'intense développement avec l'aventure spatiale, suscitent un renouveau d'intérêt tout particulier dans le domaine des usages terrestres. Bien que les motivations qui conduisent au choix du photovoltaïque comme source d'énergie soiẹnt, dans ces deux cas, relativement différentes, il existe toutefois une motivation commune qui est la notion d'autonomie. Lorsque l'on parle d'autonomie, on se réfère généralement à ce que l'on pourrait qualifier d'autonomie de lieu, mais toute aussi importante est la notion d'autonomie de fonctionnement.

Dans le cas d'utilisation de l'énergie photovoltaïque dans les satellites, ces deux aspects de l'autonomie sont bien évidemment satisfaits mais nécessitent une fiabilité absolue des composants, une complexité des commandes certaine, et en conséquence, un prix très élevé de l'ensemble. Ce dernier point limite de façon critique une extension directe des systèmes spatiaux

$\left(^{*}\right)$ Laboratoire associé au C.N.R.S. aux utilisations terrestres. Il a donc été nécessaire de reconsidérer ces problèmes, notamment dans le cas d'utilisations sous conditions climatiques difficiles. Si peu de difficultés ont été rencontrées dans le cas des générateurs de quelques watts, il n'en a pas été de même pour les systèmes de plus fortes puissances où le problème, parmi d'autres, du stockage de l'énergie électrique est très vite apparu comme un facteur limitatif sérieux. En effet, l'utilisation de batteries s'accompagne de contraintes non négligeables :

- valeur élevée du rapport masse des accumulateurs/énergie stockée,

- conditions relativement strictes des niveaux de charge et décharge si on veut conserver une durée de vie acceptable pour les batteries,

- maintenance non nulle à laquelle s'ajoute le problème du local (aération, etc.), et nous retrouvons ici la notion d'autonomie de fonctionnement,

- baisse du rendement énergétique de la chaîne,

- incidence sur le prix de l'ensemble.

Ce problème est l'un des premiers que l'on se pose lors de la conception d'un système photovoltaïque. 


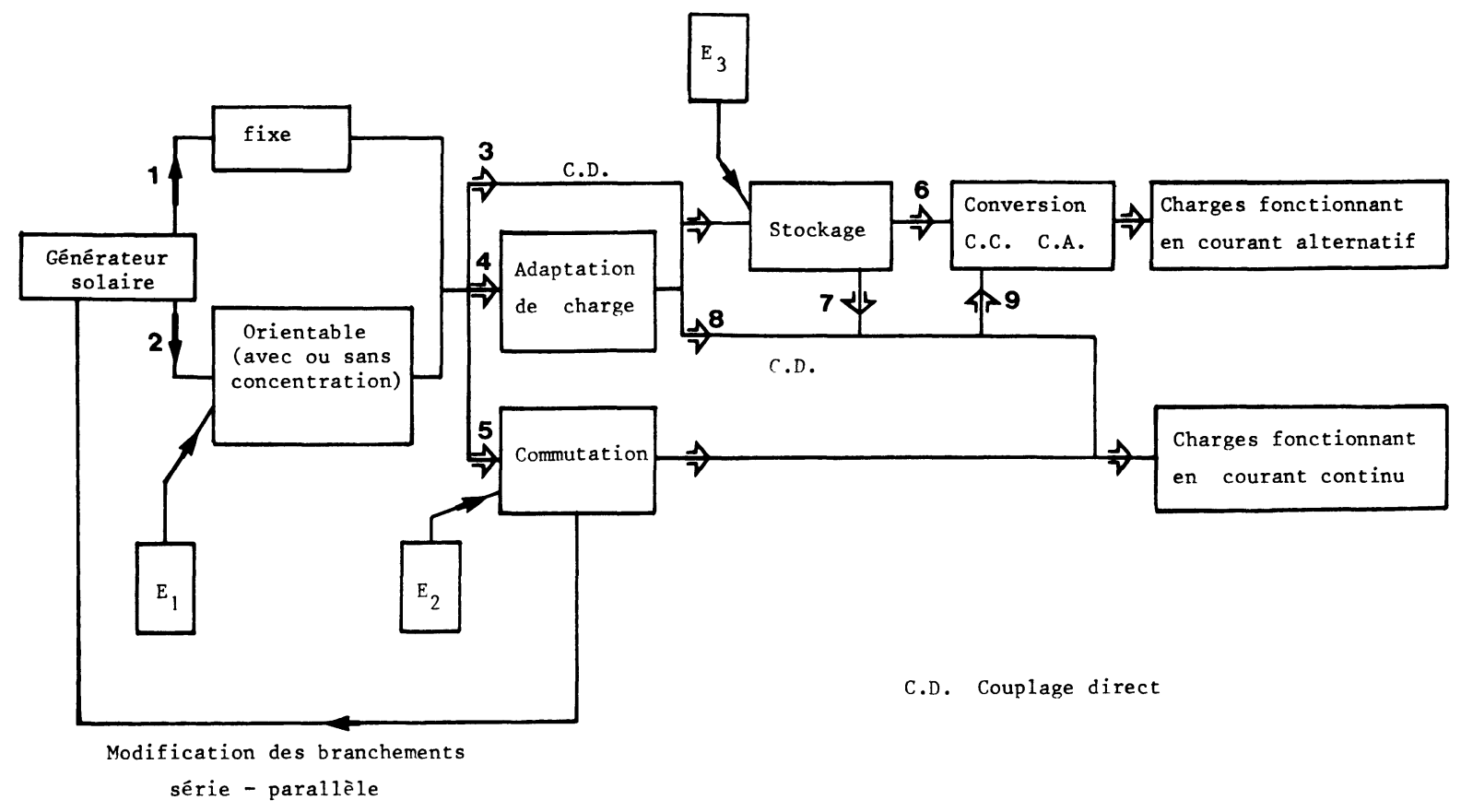

Fig. 1. - Les générateurs photovoltaïques; tableau synoptique.

[Photovoltaic generators; synoptic diagram.]

De façon générale, la figure 1 présente les variantes possibles d'un tel système :

- le générateur solaire peut être fixe (1) ou orientable (2) (orientation manuelle ou suivi du soleil par pilotage électronique $\left(E_{1}\right)$ ),

- les charges peuvent être de deux types, à courant continu ou courant alternatif.

La filière (5) correspond à la possibilité d'adapter à chaque instant le groupement de panneaux solaires à la charge par commutation série-parallèle des panneaux (électronique $\mathrm{E}_{2}$ ).

Les filières les plus utilisées sont les filières (1-3-6) et (1-3-7) où un stockage piloté par l'électronique $E_{3}$ permet d'alimenter des charges continues (1-3-7), ou des charges alternatives par l'intermédiaire d'un onduleur (1-3-6).

Nous avons mentionné plus haut les inconvénients associés au stockage de l'énergie électrique et il faut à présent noter qu'il existe des cas où ce stockage n'est pas une obligation : on peut citer le pompage de l'eau, le séchage de denrées en ventilation forcée ou la création de froid. Dans ce cas, la charge fonctionne au fil du soleil et l'autonomie de fonctionnement peut être complète même pour des puissances de quelques $\mathrm{kW}[1]$.

De tels systèmes sans stockage de l'énergie électrique sont représentés sur la figure 1 par les filières (3-8) et (3-8-9) ou (4-8) et (4-8-9). Le couplage direct (1-3-8) conduit à la plus simple des chaînes photovoltaiques, et le problème à résoudre est de concevoir l'ensemble du système de façon à extraire le maximum de puissance du générateur photovoltaïque à tous les niveaux de flux solaire $[1,2]$. Un bref rappel des conditions auxquelles doit satisfaire la charge pour atteindre un tel objectif va permettre de montrer qu'il existe des cas où l'adaptation en couplage direct ne peut être optimisée et où il faut alors faire appel à un adaptateur de charge (1-4-8). Nous nous sommes intéressés essentiellement au cas des charges continues, le cas des charges alternatives sans stockage électrique a été envisagé par exemple par Ir. C. Franx [3].

\section{Couplage direct entre panneaux solaires photo-} voltaïques et charge. - Pour extraire le maximum de puissance d'un générateur photovoltaïque il faut que le point de fonctionnement se situe dans une zone $D$ pratiquement verticale dans le diagramme couranttension du groupement (Fig. 2). Il est donc déjà évident qu'il n'est pas possible d'effectuer un couplage direct correct avec une charge purement résistive. L'adaptation ne sera bonne dans ce cas que pour une seule valeur du flux lumineux. Par contre, si l'on considère le cas des moteurs à courant continu, il est possible d'obtenir des adaptations satisfaisantes [4].

Trois types de moteurs à courant continu peuvent être envisagés : le moteur à aimants permanents, le moteur série et le moteur shunt. Le moteur à excitation séparée ne présente pas d'intérêt en raison de ce qui vient d'être dit : il n'est pas possible d'obtenir une bonne adaptation, à tous les niveaux de flux solaire, entre les panneaux utilisés pour l'excitation et l'inducteur. Nous nous restreindrons ici à l'analyse du cas du moteur à aimants permanents et préciserons les conclusions relatives aux deux autres types de moteurs [4]. 


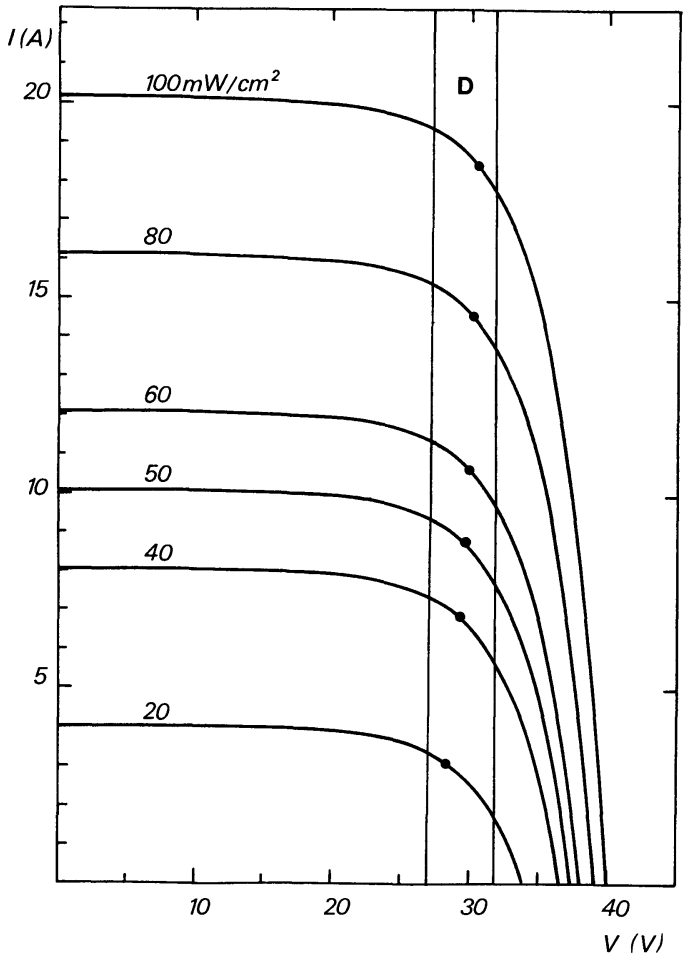

Fig. 2. - Caractéristiques courant-tension d'un groupement photovoltaique. D : zone de puissance maximale.

[Current-voltage characteristics of a photovoltaic solar array. D : maximum power zone.]

Les équations d'un moteur à aimants permanents s'écrivent :

$$
\begin{gathered}
V_{\mathrm{M}}-R_{\mathrm{i}} I_{\mathrm{M}}=K^{\prime} \varphi N \\
C_{\mathrm{E}}=K^{\prime \prime} \varphi I_{\mathrm{M}} .
\end{gathered}
$$

$I_{\mathrm{M}}$ et $V_{\mathrm{M}}$ sont le courant et la tension du moteur, donc le courant délivré par les panneaux et la tension aux bornes de ces derniers puisqu'il y a couplage direct.

$R_{\mathrm{i}}$ est la résistance d'induit, $N$ la vitesse de rotation, $\varphi$ le flux magnétique (pratiquement constant dans ce cas), $C_{\mathrm{E}}$ le couple électromécanique et $K^{\prime}$ et $K^{\prime \prime}$ deux constantes caractéristiques du moteur.

La charge caractérisée par son couple résistant $C_{\mathrm{R}}=f(N)$ définit par elle seule le point de fonctionnement. L'équation (2) fixe en effet $I_{M}$ et l'équation (1) fixe $V_{M}$. Nous obtenons ainsi pour chaque valeur de $N$, un couple de valeurs $\left(I_{\mathrm{M}}, V_{\mathrm{M}}\right)$ qui détermine la courbe de fonctionnement du système, courbe déterminée indépendamment du générateur photovoltaïque. Un exemple correspondant à un système de pompage est donné par la figure 3 [1].

Ainsi en couplage direct on peut dire que le générateur photovoltaique ne pilote pas le système. Sous un flux lumineux $\varphi$, le point de fonctionnement est obtenu par l'intersection de la courbe de fonctionnement et de la courbe de puissance du groupement de panneaux correspondant à la valeur $\varphi$. Si le flux

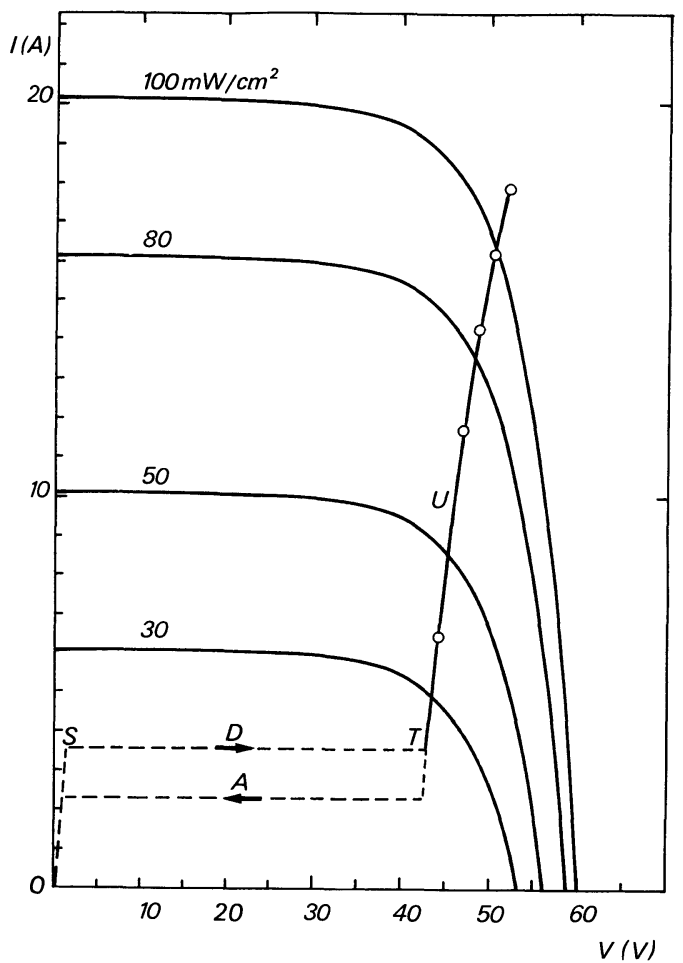

Fig. 3. - Exemple de courbe de fonctionnement en couplage direct (pompe de surface de $20 \mathrm{~m}^{3} / \mathrm{h}$ ). D : démarrage; A : arrêt.

[Example of an operating curve in a direct coupled system $\left(20 \mathrm{~m}^{3} / \mathrm{h}\right.$ surface pump). D : starting: A : stoppage.]

solaire varie, le point de fonctionnement se déplace le long de la courbe de fonctionnement. Un couple d'équations telles que (1) et (2) peut être obtenu également dans le cas des deux autres types de moteurs et le raisonnement reste le même.

Toutefois nous devons considérer le problème du démarrage, facteur important pour l'autonomie de l'ensemble. Sur générateur à tension constante, tout type de moteur continu nécessite une procédure spéciale de démarrage destinée à limiter le courant dans les bobinages. Cette limitation est automatiquement réalisée dans le cas du couplage direct sur panneaux photovoltaiques car, l'impédance du moteur étant très faible au démarrage, le générateur fonctionne en générateur de courant (région O-S, figure 3). Il n'y a donc aucun risque de surintensité dans les bobinages, et en conséquence aucune procédure spéciale n'est nécessaire; le système démarre et s'arrête seul (S-T, figure 3).

Contrairement à ce qui se passe dans le cas d'une alimentation à tension constante, c'est le moteur à aimants permanents qui démarre le mieux en couplage direct sur générateur photovoltaïque, et non le moteur série. Ceci vient du fait que dans le second cas, une partie du courant délivré par les panneaux est utilisée pour générer le champ magnétique et ne peut ainsi participer au couple de démarrage.

Le moteur shunt est à rejeter pour le couplage direct, il ne peut en effet démarrer qu'à flux solaire relativement élevé. Tout le courant généré par les 
panneaux solaires étant absorbé par l'induit, l'inducteur ne peut créer de champ magnétique suffisant.

La figure 3 montre que pour obtenir une bonne adaptation en couplage direct, deux conditions devront être satisfaites :

a) Faible couple résistant initial $C_{\mathrm{RD}}$ (pour obtenir un démarrage au flux solaire le plus faible possible) et montée rapide en vitesse de rotation.

b) Courbe de fonctionnement la plus verticale possible (région $\mathrm{D}$, figure 2).

Ces deux dernières conditions permettent de définir une charge mécanique idéale pour le couplage direct (courbe 1, figure 4) caractérisée par les points suivants [4] :

- un couple de démarrage $C_{\mathrm{RD}}$ le plus faible possible,

- une valeur de $\mathrm{d} C_{\mathrm{R}} / \mathrm{d} N$ faible pour les vitesses correspondant au démarrage,

- une valeur de $\mathrm{d} C_{\mathrm{R}} / \mathrm{d} N$ élevée pour la zone de fonctionnement.

On constate donc sur la figure 4 que les seules charges intéressantes pour le couplage direct sont les charges de type centrifuge (courbe 2, figure 4), d'où les applications pour le pompage de l'eau (avec pompe de type centrifuge) et la ventilation forcée.

Toute l'optimisation du système consistera donc à choisir les éléments de la chaîne, ou à les modifier, pour obtenir une courbe $C_{\mathrm{R}}=f(N)$ aussi proche que possible de la courbe idéale. Le groupement série-parallèle des panneaux solaires sera défini $a$ posteriori en fonction de la courbe obtenue. Un tel ajustement peut être effectué relativement rapidement,

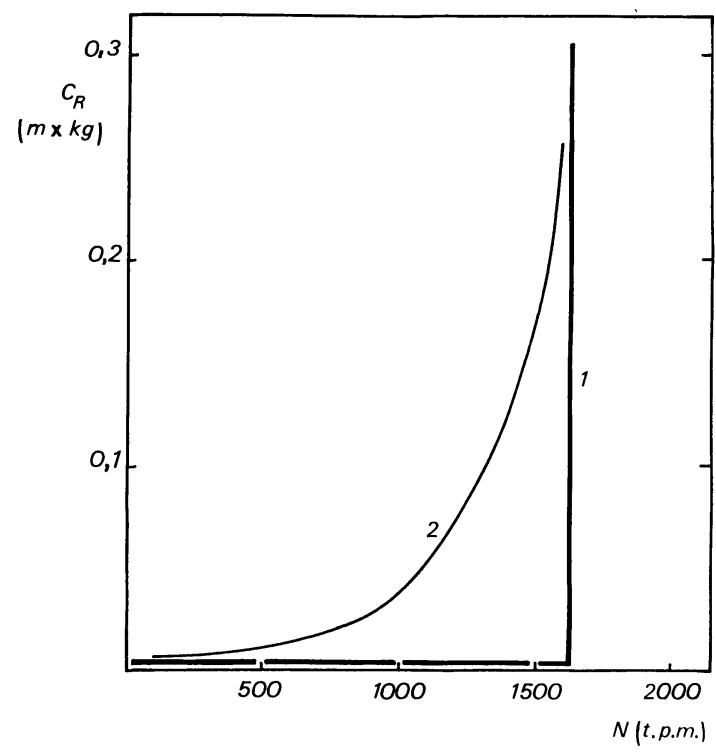

Fig. 4. - Couple résistant de la charge mécanique en fonction de la vitesse de rotation . 1 : charge idéale; 2 : charge de type centrifuge (les unités sur les axes sont données à titre d'exemple).

[Resisting torque of the mechanical load as a function of the rotation speed. 1 : ideal curve; 2 : centrifugal type load (units on axis are arbitrary).] bien qu'au coup par coup, à l'aide d'un programme de calcul [1], mais n'est hélas pas toujours réalisable. Il est alors nécessaire d'envisager un adaptateur de charge si l'on se refuse à employer un stockage électrique et si l'on veut extraire le maximum d'énergie des panneaux solaires.

3. Adaptateur de charge continu-continu. - Un exemple type où le couplage direct n'est pas réalisable est le cas d'une charge purement résistive. La figure 5 présente à titre d'exemple la courbe de charge d'un groupe frigorifique à absorption. L'intérêt d'un adaptateur de charge est de faire fonctionner les panneaux à leur point de puissance optimal, quels que soient l'ensoleillement et la charge, et de délivrer cette puissance à l'utilisation. L'adaptateur de charge permet un transfert de puissance du générateur vers la charge le long de la courbe d'équipuissance (P).

Différents types d'adaptateurs sont envisageables : ils peuvent être élévateurs ou abaisseurs de tension et leur technologie dépendra de la puissance mise en jeu. Dans le cas des faibles puissances (inférieures à $1,5 \mathrm{~kW}$ ), l'utilisation des basses tensions (quelques dizaines de volts) est encore possible au niveau du groupement de panneaux, les chutes de tension en ligne restant négligeables. Pour ces faibles puissances, une technologie à base de transistors peut être utilisée.

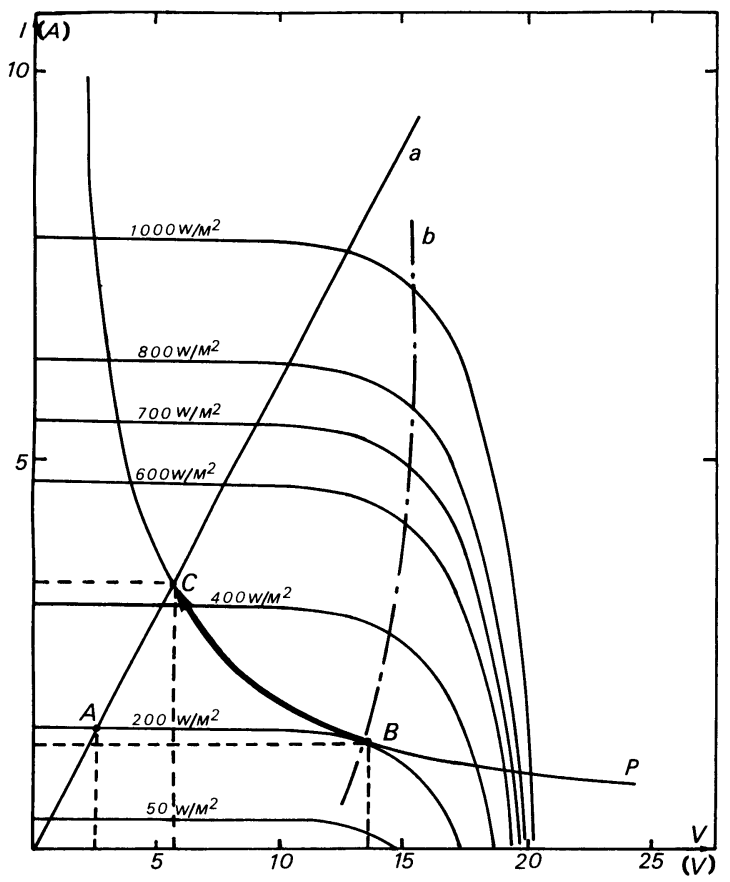

Fig. 5. - Groupement de 120 watts-crête (11 panneaux R.T.C. BPX 47A en parallèle), a : caractéristique $I=f(V)$ de charge d'un groupe frigorifique à absorption; $b$ : lieu des points de puissance maximale : $A$ : point de fonctionnement sans adaptateur: B : point de fonctionnement avec adaptateur.

[120 peak-watts array (11 panels R.T.C. BPX 47A in parallel). a : $I=f(V)$ load curve of an absorption refrigerating group; $\mathrm{b}$ : maximum power curve ; $\mathrm{A}$ : operating point without adaptator : $\mathrm{B}$ : operating point with adaptator.] 
Pour des puissances supérieures (quelques $\mathrm{kW}$ ), il est nécessaire de travailler à tension plus élevée (de l'ordre de $200 \mathrm{~V}$ ) pour ne pas être amené à un dimensionnement excessif des sections des conducteurs. Le choix entre transistors et thyristors se fera alors en fonction de critères liés au rendement énergétique. De façon générale, le choix entre un adaptateur élévateur ou abaisseur de tension est fixé par le positionnement de la caractéristique $I=f(V)$ de la charge considérée, par rapport au lieu des points de puissance maximale (courbe b, figure 5).

Quel que soit le type d'adaptateur choisi, plusieurs conditions doivent être obligatoirement remplies :

- le groupement de panneaux doit fonctionner au voisinage du point de puissance maximale quel que soit le flux lumineux,

- l'adaptateur doit être autonome, c'est-à-dire alimenté par les panneaux eux-mêmes (pas de batteries propres à l'adaptateur),

- la puissance consommée par l'adaptateur doit être la plus faible possible,

- le lieu des points de puissance maximale se déplaçant avec la température, il est nécessaire d'effectuer un suivi de cette température, celle-ci étant prélevée par une sonde sur les panneaux euxmêmes. Ce système automatique ne doit pas non plus comporter de batteries.

Nous avons mis au point deux adaptateurs de type hacheur abaisseur de tension, l'un d'une puissance maximale de $300 \mathrm{~W}$ fonctionnant sous une tension

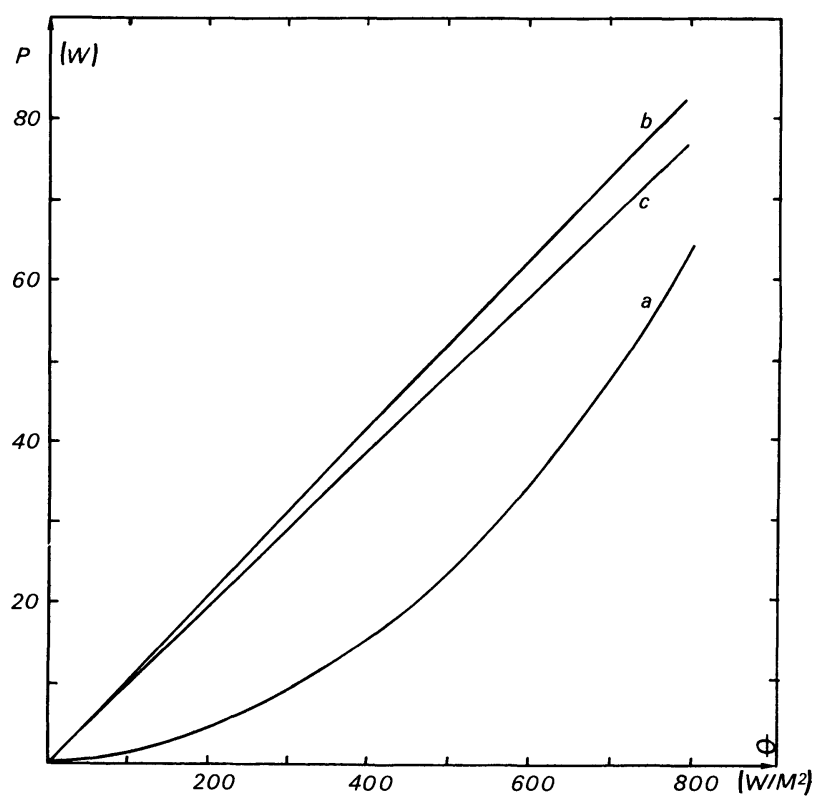

Fig. 6. - Adaptateur $300 \mathrm{~W}$ couplé au groupe frigorifique. a : puissance transmise à la charge en couplage direct $P_{\mathrm{CD}} ; \mathrm{b}:$ puissance $P_{\mathrm{EA}}$ à l'entrée de l'adaptateur; $\mathrm{c}$ : puissance transmise à la charge par l'adaptateur $P_{\mathrm{SA}}$ en fonction de l'ensoleillement.

[300 W adaptator coupled with the refrigerating group. a : power transmitted to the load in direct coupling $P_{\mathrm{CD}} ; \mathrm{b}$ : power $P_{\mathrm{EA}}$ at adaptator input : $\mathrm{c}$ : power transmitted to the load through adaptator $P_{\mathrm{SA}}$ as a function of light flux.] de $15 \mathrm{~V}$, l'autre d'une puissance maximale de $1,5 \mathrm{~kW}$, fonctionnant sous une tension de $30 \mathrm{~V}$. Toute la technologie est à base de transistors et les composants ont été spécialement choisis pour minimiser les pertes. Cet adaptateur fonctionne à fréquence variable (fonction du flux lumineux) et ne nécessite aucune source, de tension référence, extérieure [5].

Nous présentons ci-dessous les résultats obtenus avec ces deux adaptateurs sur deux types de charges. Le premier cas correspond à la charge purement résistive présentée précédemment (figure 5). La deuxième charge est constituée par un système de pompage d'eau alimenté par panneaux photovoltaiques en couplage direct, et installé en Corse [1].

Le groupement de onze panneaux en parallèle alimentant le bloc frigorifique délivre une puissance crête de $120 \mathrm{~W}$. La figure 6 présente les résultats obtenus en fonction de l'ensoleillement. L'énergie fournie à la charge étant transmise sous forme hachée, sa mesure précise a nécessité la réalisation d'un wattmètre instantané. Il est possible de définir un gain $G$ de l'adaptateur par le rapport de la puissance cédée à la charge avec adaptateur, à la puissance cédée à cette même charge sans adaptateur, et ceci pour chaque valeur de l'ensoleillement. La figure 7 présente la variation de ce gain $G$ avec le flux solaire et les valeurs obtenues soulignent tout l'intérêt de l'utilisation d'un adaptateur de charge. Le rendement peut être défini comme le rapport entre la puis-

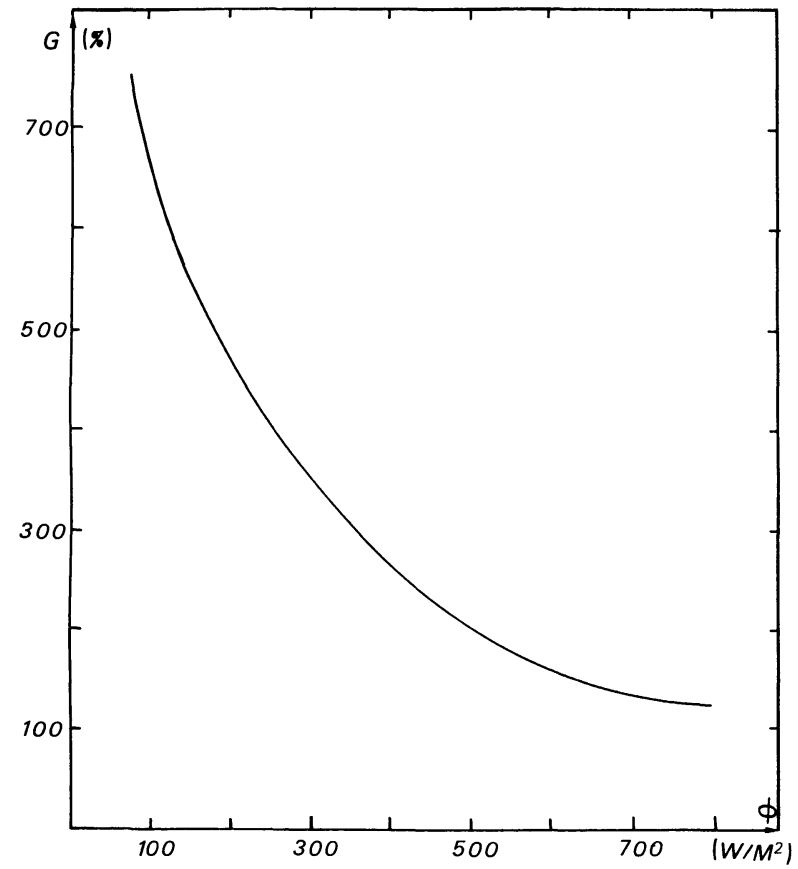

Fig. 7. - Adaptateur $300 \mathrm{~W}$. Variation du gain $G$ (rapport de la puissance $P_{\mathrm{SA}}$ transmise à la charge avec adaptateur, à la puissance $P_{\mathrm{CD}}$ transmise à la même charge sans adaptateur) en fonction de l'ensoleillement.

[ $300 \mathrm{~W}$ adaptator. Variation of gain $G$ (ratio of power $P_{\mathrm{SA}}$ transmitted to the load through adaptator, to power $P_{\mathrm{CD}}$ transmitted to the same load without adaptator) as a function of light flux.] 
sance à la sortie et la puissance à l'entrée d'un tel appareil. Dans ce cas d'adaptateur de très faible puissance (300 W maximum), ce rendement $\eta$ varie suivant la puissance transférée de $90 \%$ à $98 \%$.

La figure 8 présente les caractéristiques de l'ins tallation de pompage située en Corse. Elle est constituée de 56 panneaux BPX 47 A délivrant une puissance de $600 \mathrm{~W}$ crête. Il est à noter que pour un flux de $580 \mathrm{~W} / \mathrm{m}^{2}$, et pour des valeurs supérieures, l'adaptateur, qui est du type abaisseur de tension, doit se comporter comme un court-circuit.

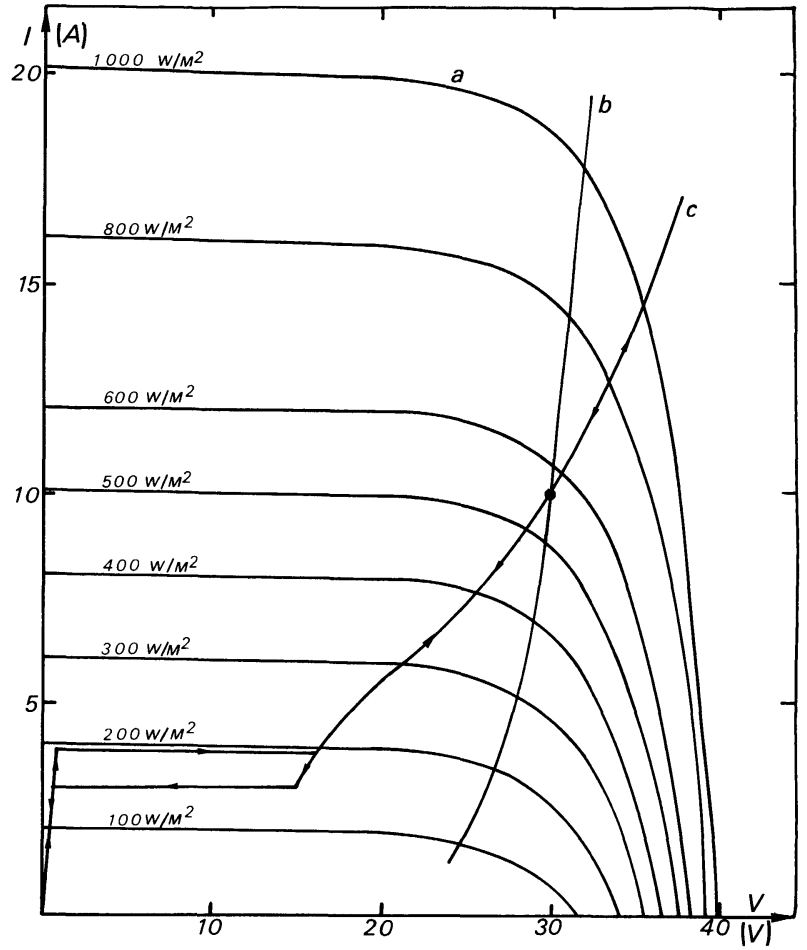

Fig. 8. - Système de pompage d'eau de 600 watts-crête. a : courbes caractéristiques du groupement à $25^{\circ} \mathrm{C} ; \mathrm{b}$ : lieu des points de puissance maximale; c : courbe de fonctionnement de la charge.

[600 peak-watts water pumping system. a : characteristics of the array at $25^{\circ} \mathrm{C} ; \mathrm{b}$ : maximum power curve; c : operating curve of the load.]

Donc dans cet exemple précis, son action ne sera effective que pour des flux inférieurs à $580 \mathrm{~W} / \mathrm{m}^{2}$. Les résultats obtenus avec l'adaptateur de puissance maximale $1,5 \mathrm{~kW}$ couplé à la moto-pompe décrite précédemment, sont ainsi portés sur la figure 9. Nous vérifions bien l'intérêt de l'adaptateur pour des flux inférieurs à $580 \mathrm{~W} / \mathrm{m}^{2}$ et son inutilité au-dessus de cette valeur. Nous avons porté sur la figure 10 le rendement propre $\eta$ de l'appareil; la diode de protection placée en série avec le groupement de panneaux n'entre pas en ligne de compte, étant normalement installée qu'il y ait ou non un adaptateur. Le rendement $\eta$ dépasse $90 \%$ dès que la puissance transmise est de l'ordre de $80 \mathrm{~W}$ et atteint $98 \%$ pour une puissance transmise de $300 \mathrm{~W}$.

Ces mesures sur un système en grandeur réelle

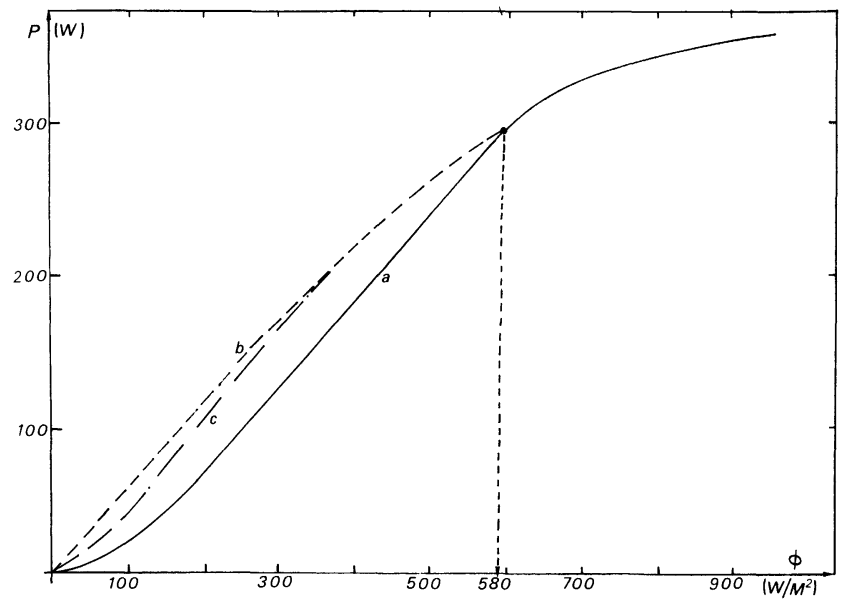

Fig. 9. - Adaptateur 1,5 kW couplé au système de pompage d'eau. a : puissance transmise à la charge en couplage direct $P_{\mathrm{CD}} ; \mathrm{b}$ : puissance $P_{\mathrm{EA}}$ à l'entrée de l'adaptateur; c : puissance transmise à la charge par l'adaptateur $P_{\mathrm{SA}}$ en fonction de l'ensoleillement.

[1.5 $\mathrm{kW}$ adaptator coupled with the water pumping system. a : power transmitted to the load in direct coupling $P_{\mathrm{CD}} ; \mathrm{b}:$ power $P_{\mathrm{EA}}$ at adaptator input; $\mathrm{c}:$ power transmitted to the load through adaptator $P_{\mathrm{SA}}$ as a function of light flux.]

nous ont permis de contrôler la fiabilité de l'adaptateur élaboré et d'en déterminer les caractéristiques, telles que consommation intrinsèque, gain et rendement. Malheureusement, dans le cas de nos expériences sur le prototype de Corse, il ne nous était pas possible de modifier le groupement de panneaux, ce qui nous

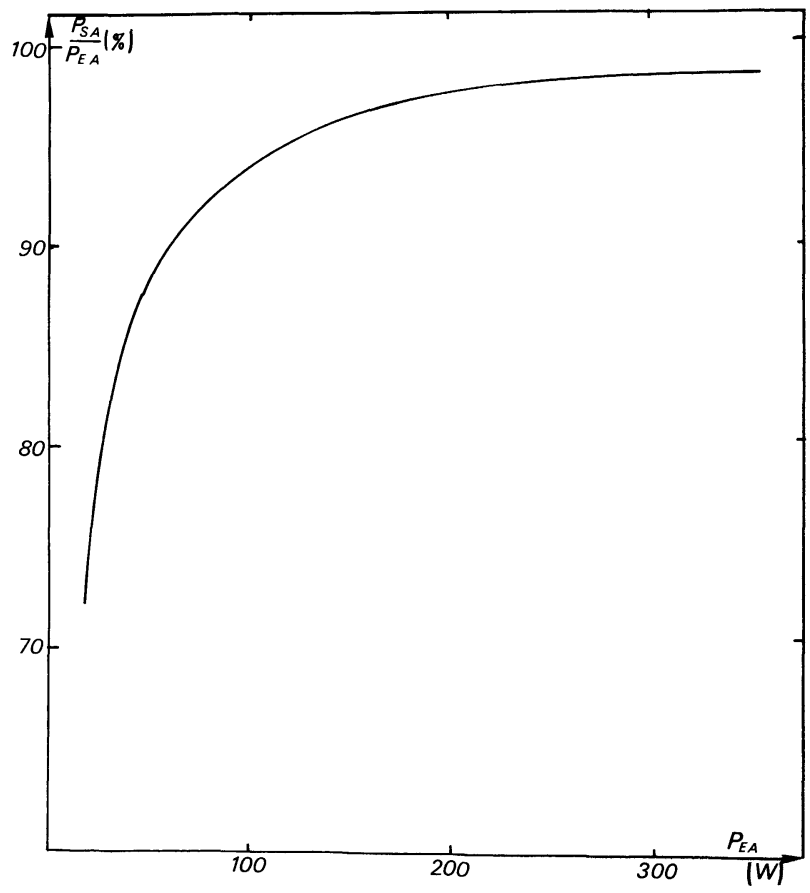

Fig. 10. - Rendement de l'adaptateur 1,5 kW (rapport des puissances à la sortie $P_{\mathrm{SA}}$ et à l'entrée $P_{\mathrm{EA}}$, de l'adaptateur) en fonction de la puissance à transmettre.

[Efficiency of the $1.5 \mathrm{~kW}$ adaptator (ratio of powers at output $P_{\mathrm{SA}}$ and input $P_{\mathrm{EA}}$ of the adaptator) as a function of power.] 
aurait permis de positionner la courbe de fonctionnement de la moto-pompe (courbe c, figure 9) entièrement à gauche de la courbe b et de tester ainsi l'appareil sur toute la gamme de puissance disponible (jusqu'à $600 \mathrm{~W}$ ). Il aurait alors fallu modifier aussi la tension de fonctionnement de l'adaptateur.

4. Conclusion. - L'alimentation de charges de type continu par générateurs photovoltaïques ne nécessite pas obligatoirement l'utilisation de batteries et nous avons montré qu'il est alors possible d'élaborer des ensembles, soit à couplage direct, soit utilisant un adaptateur de charge, pour lesquels pratiquement toute l'énergie disponible peut être extraite des panneaux solaires.

Dans le cas du couplage direct panneaux-moteur continu, les charges mécaniques de type centrifuge conduisent à de très bonnes adaptations moyennant un choix approprié des éléments de la chaîne.

L'adaptateur de charge quant à lui permet d'obtenir une adaptation rigoureuse sur n'importe quel type de charge électrique, se traduisant par des rendements situés entre 96 et $98 \%$ moyennant la réalisation d'un suivi en température. Etant alimenté par le groupement photovoltaïque lui-même, l'adaptateur permet une autonomie totale de fonctionnement équivalente à celle obtenue dans le cas du couplage direct. De plus, son utilisation permet de diminuer le nombre de panneaux photovoltaïques pour une puissance fournie déterminée.

Remerciements. - L'étude des adaptateurs de charges décrits dans cet article a été effectuée grâce à un contrat C.O.M.E.S. no 78031 dont une partie a fait l'objet des travaux de fin d'études de Messieurs B. Canut et E. Descours, étudiants de troisième année de l'Institut de Chimie et Physique Industrielles de Lyon (I.C.P.I.). Nous remercions Monsieur G. Barreault, Chef de Service Electronique de 1'I.C.P.I. ainsi que Monsieur J. P. Gelin, Professeur à l'I.C.P.I., pour leurs précieux conseils.

\section{Bibliographie}

[1] Roger, J. A. et al., Proc. 1977 Photovoltaic Solar Energy Conf., p. 1211, Reidel, Boston (1977).

[2] Appelbaum, J., Bany, J., Proc. 1977 Photovoltaic Solar Energy Conf., p. 1243, Reidel, Boston (1977).
[3] Franx, Ir. C., Proc. 1979 Photovoltaic Solar Energy Conf., p. 1038, Reidel, Boston (1979).

[4] Roger, J. A., J. Sol. Energy 23 (1979) 193-198.

[5] Barlaud, M. et Masselot, C., Proc. 1977 Photovoltaic Solar Energy Conf., p. 779, Reidel, Boston (1977). 